\title{
HIGH-RESOLUTION 22 GHZ CONTINUUM OBSERVATIONS OF NGC 4258
}

\author{
Implications for Advection-Dominated Accretion
}

\author{
J.R. HERRNSTEIN \\ National Radio Astronomy Observatory \\ PO Box 0, Socorro NM, 87801
}

VLBA observations of the water megamaser in NGC 4258 reveal an extremely thin, nearly edge-on, slightly warped disk in Keplerian rotation around a $3.5 \pm 0.1 \times 10^{7} \mathrm{M}_{\odot}$ central mass (Miyoshi et al. 1995). The masers extend from 0.13-0.26 pc (assuming a distance of $6.4 \mathrm{Mpc}$ ), and provide an unusual opportunity to probe active galactic nuclei (AGN) accretion on sub-parsec scales and in a system where the mass of the central engine and the geometry of the outer accretion disk are known with precision. Here, I discuss new $22 \mathrm{GHz}$, VLBA continuum observations of NGC 4258 in the context of advection-dominated accretion models. A more detailed discussion is in preparation (Herrnstein et al. 1998).

The disk binding mass corresponds to an Eddington luminosity of $4.5 \pm$ $0.1 \times 10^{45} \mathrm{erg} \mathrm{s}^{-1}$. The NGC 4258 bolometric luminosity is estimated from ASCA X-ray data (Makishima et al. 1994) and optical polarimetry (Wilkes et al. (1995) to be $\sim 10^{42 \pm 1}$. Thus, NGC 4258 is highly sub-Eddington, and there are two basic models to explain this. The first is that a mass-starved, Shakura-Sunyaev thin accretion disk extends all the way to the central engine. Neufeld \& Maloney (1995) have argued that the extremely low accretion rates $\left(\sim 10^{-4} \alpha \mathrm{M}_{\odot} \mathrm{yr}^{-1}, \alpha\right.$ being the standard Shakura-Sunyaev parameterization of the kinematic viscosity) required by this model are consistent with the observed radial extent of the maser emission. An alternative model is that NGC 4258 harbors an optically thin, two-temperature, advection-dominated accretion flow (ADAF; Narayan \& Yi 1995) in which the majority of energy released through viscous dissipation is carried through the central event horizon by a very hot $\left(\sim 10^{12} \mathrm{~K}\right)$ ion plasma. Lasota et al. (1996; hereafter L96) fit the NGC 4258 optical-to-X-ray spectrum with a central ADAF accreting at $\sim 10^{-1.9} \alpha \mathrm{M}_{\odot} \mathrm{yr}^{-1}$. In comparison to standard thin-disk models, the ADAF is thus very rapid and very inefficient. 
In the ADAF models, the hot ion plasma co-exists with cooler $(\sim$ $10^{9.5} \mathrm{~K}$ ) electrons that are expected to generate significant radio synchrotron emission. L96 predict a flux density of about $5 \mathrm{mJy}$ at $22 \mathrm{GHz}$, and we have searched for this emission in a multi-epoch VLBI experiment including the VLBA, phased VLA, and Greenbank 140-foot of the NRAO, and the Efflesburg 100-meter telescope. The masers pinpoint the position of the disk center to better than 0.1 mas (Herrnstein 1997), and there is no ambiguity about where the ADAF emission should occur. We detect no central-engine $22 \mathrm{GHz}$ continuum emission in $N G C 4258$ at the level of $220 \mu \mathrm{Jy}(3 \sigma)$.

Broadband ADAF spectra have been applied successfully to a number of systems, ranging from Galactic X-ray binaries to AGN (see Narayan 1997 for a review). While the present radio data are inconsistent with the models of L96, they cannot rule out ADAF models altogether in NGC 4258. The outer accretion flow in NGC 4258, as traced by the masers, is evidently cool and thin and the transition to a geometrically thick ADAF must occur somewhere within $0.13 \mathrm{pc}\left(\sim 4 \times 10^{4} \mathrm{Sch}\right.$ warzschild radii, $\left.\mathrm{R}_{S}\right)$. The predicted $22 \mathrm{GHz}$ emission can be suppressed by postulating that this transition occurs within the radius at which most of the ADAF 22 $\mathrm{GHz}$ emission is generated. For the NGC $4258 \mathrm{ADAF}$, this is about $10^{2}$ $\mathrm{R}_{S}$ (c.f. Mahadevan 1997), and the present upper limit on the NGC 4258 central-engine radio emission requires that any central ADAF must be truncated within $\sim 10^{2} R_{S}$. Rigorous numerical simulations confirm this result (Narayan, personal communication). More sensitive radio observations will either detect the ADAF emission, or place progressively tighter geometric constraints on the models.

\section{References}

Herrnstein, J. R., PhD Dissertation, Harvard University, 1997 (H97).

Herrnstein, J. R., Greenhill, L. J., Moran, J. M., Diamond, P. J., Inoue, M., Miyoshi, M., and Nakai, N. 1998, in preparation.

Lasota, J.-P., Abramowicz, M. A., Chen, X., Krolik, J., Narayan, R., \& Yi, I. 1996, Ap. J., 462, 142 (L96).

Mahadevan, R. 1997, Ap. J., 477, 585 (M97).

Makishima, K., Fujimoto, R, Ishisaki, Y., Kii, T., Lowenstein, M., Mushotzky, R., Serlemitsos, P., Sonobe, T., Tashiro, M., \& Yaqoob, T. 1994, Proc. Astron. Soc. Jpn., 46, L77.

Miyoshi, M., Moran, J. M., Herrnstein, J. R., Greenhill, L. J., Nakai, N., Diamond, P. J., \& Inoue, M. 1995, Nature, 373, 127.

Narayan, R. 1997, in Accretion Phenomena and Related Outflows, eds. D. Wickramasinghe, G. Bicknell, \& L. Ferrario, 75.

Narayan, R. \& Yi, I. 1995, ApJ, 452, 710.

Neufeld, D. A. \& Maloney, P. R. 1995, ApJ. (Letters), 447, L17.

Wilkes, B. J., Schmidt, G. D., Smith, P. S., Mathur, S., \& McLeod, K. K. 1995, Ap. J. (letters), 455, L13. 